\title{
A modular approach for the topological synthesis of geared robot manipulators
}

\author{
Dar-Zen Chen *, Chia-Pin Liu, Dong-Wen Duh \\ Department of Mechanical Engineering, National Taiwan University, 10617 Taipei, Taiwan
}

Received 8 August 2001; received in revised form 8 July 2002; accepted 8 July 2002

\begin{abstract}
A methodology for the modular design of geared robot manipulators (GRMs) is presented. This approach is based on that the kinematic structure of a GRM can be hierarchically decomposed into mechanical transmission lines (MTLs), and input and transmission units. The admissible units are further categorized into modular groups according to their number of links and kinematic characteristics, in which the unit with the least number of links is referred to as a basic unit. Thus, with a given configuration, GRMs can be created from basic MTLs first which contains only basic units, and then modified structures can be obtained by replacing one or more contained units according to specified kinematic requirements. This approach allows the designer to obtain proper mechanical structures to achieve a desired kinematic behavior on the base of the simplest format during the conceptual design stage.
\end{abstract}

(c) 2002 Elsevier Science Ltd. All rights reserved.

\section{Introduction}

The kinematic structure of a robotic manipulator often takes the form of an open-loop configuration. An open-loop robotic manipulator is mechanically simple and easy to construct. However, it does require the actuators to be located along the joint axes which, in turn, degrades the dynamic performance of the system. Thus, many robot manipulators are constructed in a partially closed-loop configuration to reduce the inertia loads on the actuators. For the case of geared robot manipulators (GRMs), gear trains are used to permit the actuators to be located as close to the base as possible.

\footnotetext{
* Corresponding author. Fax: +886-2-2363-1755.

E-mail address: dzchen@ccms.ntu.edu.tw (D.-Z. Chen).
} 
Several approaches for the topological synthesis of GRMs have been developed based on graph theory. Lin and Tsai [1], based on the results of 2 degree-of-freedom (DOF) non-fractionated geared kinematic chains (GKCs), enumerated the atlas of bevel-gear type spherical wrist mechanisms with up-to-eight links. Chang and Tsai [2] used the concept of mechanical transmission lines (MTLs) to describe the relations between joint torques and actuator torques in a GRM. They also generated admissible structure matrices to describe the coupling relationships between MTLs. Chen and Shiue [3] established the characteristics of input and transmission units that construct an MTL and identified input and transmission units from the atlas of non-fractionated GKCs [4-7]. With the atlas of admissible units, an approach to compose these units into MTLs and GRMs is developed accordingly by the number of links [11]. However, this method did not take kinematic properties of individual unit into consideration and thus provided no modular information about the units.

In this paper, an approach for the modular design of GRMs will be presented. Admissible input and transmission units [3] will be classified into basic and extended units according to their number of links and also be classified into ordinary and planetary units according to their kinematic characteristics. With the principle of composition, basic MTLs are constructed exclusively with basic units. Hence, in the conceptual design stage, basic GRMs can be enumerated first by composing basic MTLs according to the given configuration. Modified structures are then obtained by replacing the contained basic unit(s) with extended unit(s) in the modular groups according to the kinematic requirements. Thus, the designer can start from the simplest mechanical structure to more complex ones during the conceptual design stage. This approach leads to an effective modular design of GRMs.

\section{Hierarchical decomposition of geared robot manipulators}

\subsection{AGM level}

The functional representation of a 3-DOF GRM is shown in Fig. 1(a), in which links 1, 4, 6 are input links and link 3 is the end-effector. In the graph representation, links are denoted by vertices, joints by edges, turning pairs by thin edges, and gear pairs by heavy edges. The thin edges are labeled according to their axis locations in space. By rearranging coaxial revolute joints, a unique canonical graph representation [8] can be obtained such that all edges lying on a thin-edged path traced from the base to any other vertex have different edge labels. Fig. 1(b) shows the canonical graph representation of Fig. 1(a). Among these thin-edge paths, the linkage that starts from the base link and ends at the output link is defined as the equivalent open-loop chain (EOLC) [8]. Links on the EOLC are referred to as primary links, and all other links are called secondary links [9]. The relative angular displacement between any two adjacent primary links is referred to as a joint angle.

\subsection{Mechanical transmission line level}

The arrangement of secondary links in the GRM forms the MTLs which describe where the input actuators are located and how the input torques are transmitted to various joints. Through 


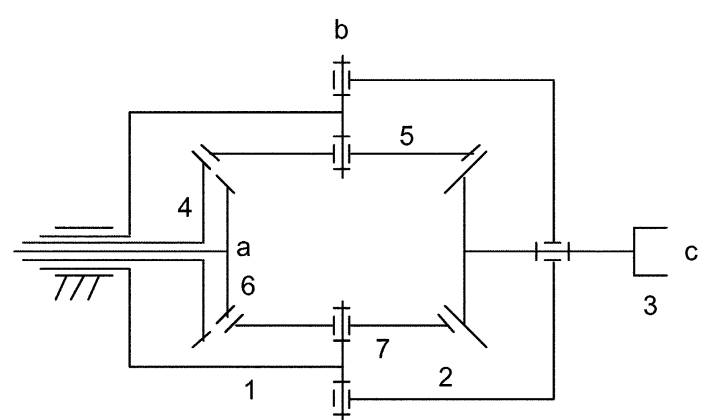

(a)

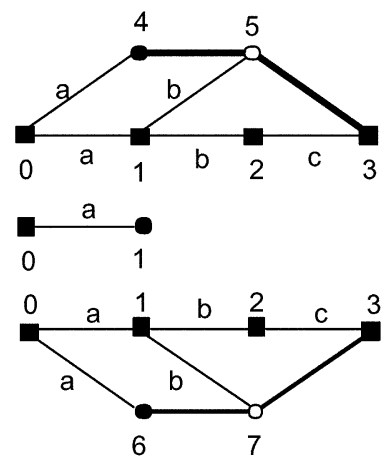

(c)

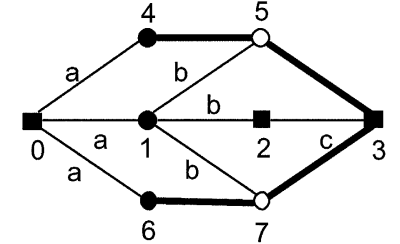

(b)

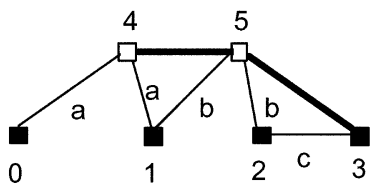

(d)

Fig. 1. A 3-DOF GRM: (a) functional representation, (b) graph representation, (c) MTLs, (d) pseudo-isomorphic graph of an MTL.

the MTL, torques are transmitted to the end-effector on which payload can be attached. By collecting the vertices and edges associated with an MTL, a graph that represents the MTL can be identified. For decoupled MTLs, the graph of a GRM can be separated into various MTL subgraphs with the EOLC as their common linkage. Fig. 1(c) shows the graph representation of the three MTLs of the example GRM. In Fig. 1(c), the two-link MTL can be regarded as a directdrive MTL, and the other MTLs are 3-joint MTLs in which input torques are exerted respectively on secondary links 4 and 6 . In the paper, we will concentrate on GRMs with decoupled MTLs.

\subsection{Unit level}

By coaxial re-arrangement, a pseudo-isomorphic graph representation [1] corresponding to the upper MTL in Fig. 1(c) can be obtained as shown in Fig. 1(d). It can be observed from Fig. 1(d) that an MTL is composed of serially connected non-fractionated kinematic chains with cut links in between.

The first non-fractionated kinematic chain associated with an MTL is referred to as the input unit and the others are called transmission units [3]. Each input unit contains an input link and 


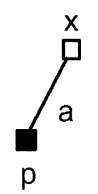

$\mathrm{UU}-1$

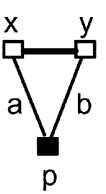

TU-1

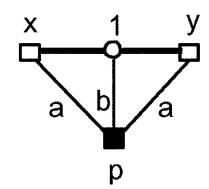

IU-2

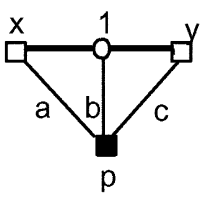

TU-2

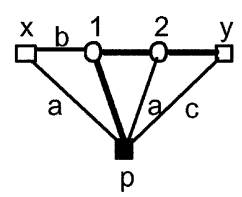

TU-6 *

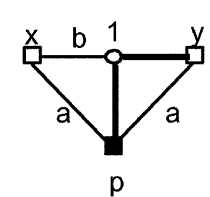

IU-3*

(a)

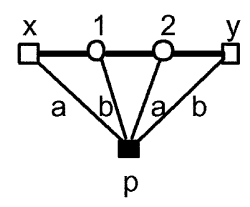

TU-3

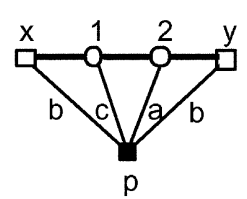

$\mathrm{IU}-4$

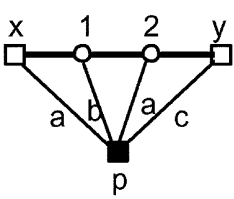

$\mathrm{TU}-4$ *

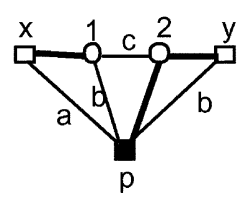

TU-7 *

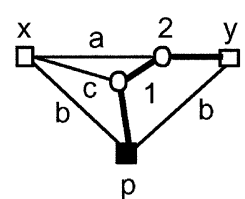

IU-5*

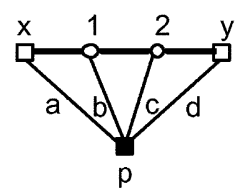

TU-5

(b)

Fig. 2. Admissible units: (a) input units, (b) transmission units.

each transmission unit is used to increase the number of joints influenced by the input. Fig. 2 shows the atlas of admissible input and transmission units with up-to-five links [3], in which IU stands for the input unit and TU stands for the transmission unit. In Fig. 2, the unit with the least number of links is called basic unit, while others are called extended units. Hence, IU-1 and TU-1 represent the basic input unit and the basic transmission unit, respectively. In Fig. 2, each primary link is represented by a solid rectangle, connecting link is represented by a hollow rectangle, and secondary link is represented by a circle. Also, in Fig. 2, the unit with an asterisk can be flipped to form a different unit, i.e., a different unit can be obtained by exchanging the roles of the post- and the pre-connecting links.

\section{Kinematic properties of units}

The pre-connecting link of a unit is considered the local input while the post-connecting link is the local output of the unit. Let links $j$ and $k$ form a gear pair and link $i$ be the carrier. Then, links $i, j$ and $k$ and their connecting joints form a fundamental circuit and the fundamental circuit equation [4] can be written as:

$$
q_{j, i}=e_{k, j} q_{k, i}
$$


where $q_{j, i}$ is the relative displace of link $j$ with respect to $i$, and $e_{k, j}= \pm N_{k} / N_{j}$ denotes the gear ratio, positive or negative varies according to a positive rotation of gear $k$ results in a positive or negative rotation of gear $j$ about their pre-defined axes of rotation, and $N_{k}$ is the teeth number of gear $k$.

Applying Eq. (1) to IU-2, the associated fundamental circuit equations can be obtained as

$$
\begin{aligned}
& q_{x, p}=e_{1, x} q_{1, p} \\
& q_{1, p}=e_{y, 1} q_{y, p}
\end{aligned}
$$

Let link $x$ be the pre-connecting link and link $y$ be the post-connecting links. By substituting Eq. (3) in Eq. (2), the angular displacement between the local input $x$ and primary link $p, q_{x, p}$, can be represented as a function of the angular displacement between local output $y$ and primary link $p, q_{y, p}$, as

$$
q_{x, p}=e_{y, 1} e_{1, x} q_{y, p}=g_{\mathrm{IU}-2} q_{y, p}
$$

where $g_{\mathrm{IU}-2}$ is called the forward gain associated with IU-2 [10].

Table 1 shows the forward gains of the admissible input and transmission units with up-to-five links. For a flippable unit, the forward gain of an operating mode is simply the reciprocal of the alternative operating mode. For instance, by choosing link $y$ in TU-7 as the local input and link $x$ as the local output, the relation between $q_{y, p}$ and $q_{x, p}$ can be written as

$$
q_{y, p}=\frac{\left(1-e_{p, 2} e_{2, y}\right)}{e_{1, x}} q_{x, p}=\frac{1}{g_{\mathrm{TU}-7}} q_{x, p}
$$

Units can be further classified according to their kinematic structures. A unit is referred to as an ordinary-type (O-type) unit if the contained primary link is the unique carrier while those units with more than one carrier are called planetary-type (P-type) unit. From Table 1, it can be seen that the forward gains associated with O-type units, $g_{\mathrm{OU}}$, and the forward gains associated with Ptype units, $g_{\mathrm{PU}}$, can be written in general as

$$
g_{\mathrm{OU}}=\Pi e
$$

and

$$
g_{\mathrm{PU}}=\prod_{i} e_{i} /\left(1-\prod_{j} e_{j}\right)
$$

From Eq. (6), it can be seen that an O-type unit is suitable for relocating the actuator or adjusting center distance between joint axes. On the other hand, Eq. (7) indicates that by proper gear train design, a P-type unit can provide pretty high forward gain which facilitates torque amplification. Hence, the O-type and P-type units represent different modular groups with distinct kinematic behaviors. Note that the two-link input unit IU-1 is classified as an O-type unit. 
Table 1

Forward gains of admissible units

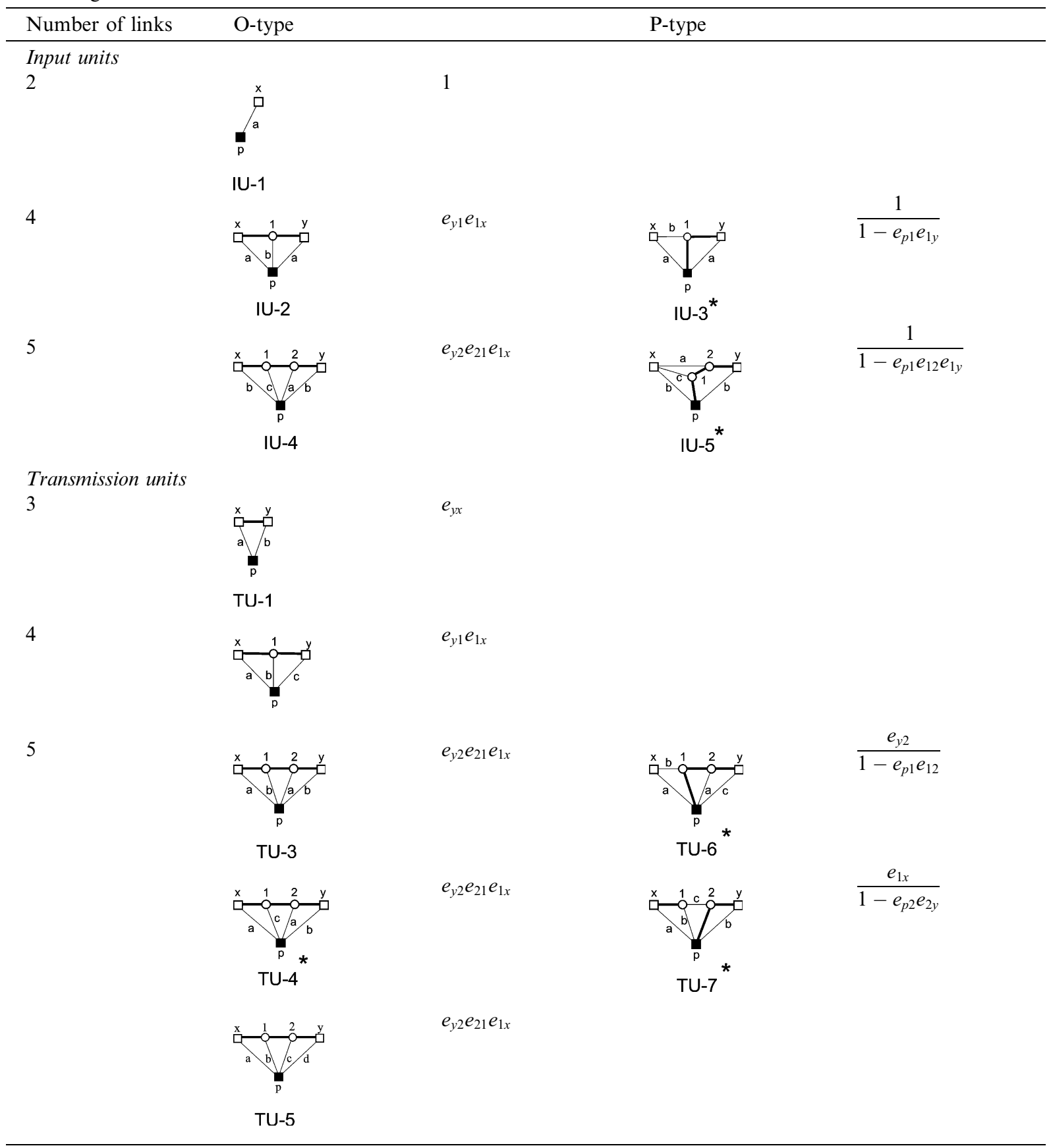




\section{Composition of mechanical transmission lines}

According to the structural hierarchy, an $n$-joint MTL is composed of one input unit and $n-1$ transmission units as shown in Fig. 3. With selected units, the general steps to compose an MTL can be broadly described as follows:

S1: Assign the pre-connecting link of the input unit as the input link. For IU-1, the one and only one connecting link is the input link.

S2: Connect each pair of adjacent units by combining the post-connecting link of the preceding unit and the pre-connecting link of the succeeding unit.

S3: Re-arrange coaxial revolute joints between the connecting link and primary links of each pair of adjacent units to form the articulated joints on the EOLC.

S4: Assign the post-connecting link of the last transmission unit as a primary link to form the last articulated joint.

From Fig. 1(d), it can be seen that input unit IU-1 shares its post-connecting link 4 to connect the pre-connecting link 4 of transmission unit TU-1. Also, secondary link 5 acts as the postconnecting and pre-connecting links of the succeeding transmission units.

Note that there are $n-1$ connecting links existing in an $n$-joint MTL. Hence, the total number of links of an $n$-joint MTL, $m_{\mathrm{m}}$, can be written as:

$$
m_{\mathrm{m}}=m_{\mathrm{IU}}+\left.\sum_{j=2}^{n} m_{\mathrm{TU}}\right|_{j}-(n-1)
$$

where $m_{\mathrm{IU}}$ is the number of links of the input unit and $\left.m_{\mathrm{TU}}\right|_{j}$ is the number of links of the $j$ th transmission unit.

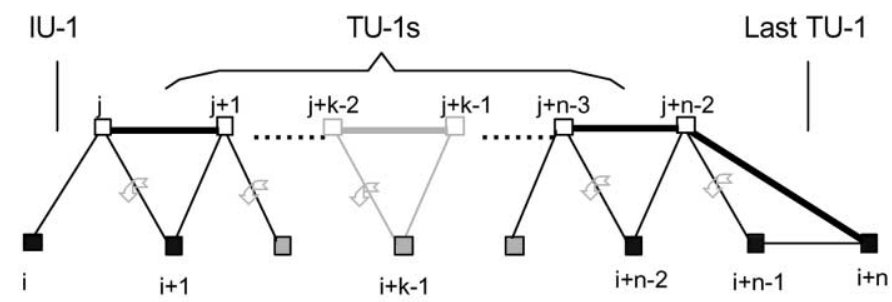

(a)
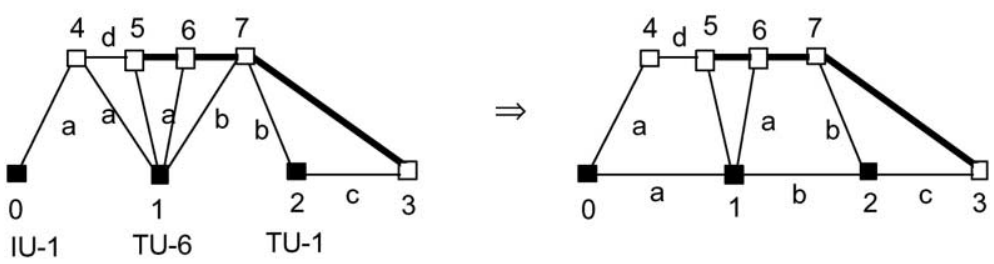

(b)

Fig. 3. Composition of an MTL: (a) a basic MTL, (b) an extended MTL. 


\subsection{Basic mechanical transmission line}

An MTL with only basic units is called the basic MTL, which has the simplest kinematic structure. The unit arrangement of a basic $n$-joint MTL can be generally expressed as

$$
\text { [IU-1, TU-1, TU-1, ., TU-1] }
$$

Fig. 3(a) shows the pseudo-isomorphic graph representation of an $n$-joint basic MTL. From Table 1, it is clear that the number of links of a basic input unit is two and the number of basic transmission units is three. Hence, Eq. (8) can be applied to basic MTLs as

$$
\left.m_{\mathrm{m}}\right|_{\mathrm{b}}=2+3(n-1)-(n-1)=2 n
$$

where $\left.m_{\mathrm{m}}\right|_{\mathrm{b}}$ is the total number of links of a $n$-joint basic MTL.

From Eq. (9), it can be determined that $\left.m_{\mathrm{m}}\right|_{\mathrm{b}}$ is equal to 2, 4, and 6 for 1-, 2- and 3-joint basic MTLs, respectively.

\subsection{Extended mechanical transmission lines}

An MTL which has at least one extended unit is called an extended MTL. An extended MTL can be obtained by replacing basic unit(s) in a basic MTL with an extended unit. The general guidelines of unit replacement may be broadly outlined as:

1. For relocating an actuator: This can be achieved by replacing the basic IU, IU-1, with an extended O-type IU, such as IU-2 or IU-4.

2. For adjusting the center distance between joint axes: This can be achieved by replacing the basic TU, TU-1, with an extended O-type TU such as TU-2, TU-3, TU-4 or TU-5.

3. For higher gain: This can be achieved by replacing the basic IU, IU-1, with an extended P-type IU such as IU-3 or IU-4, and/or by replacing the basic TU, TU-1, with an extended P-type TU such as TU-6 or TU-7.

An extended MTL with at least one P-type unit is called a P-type extended MTL while an extended MTL with only O-type units is called an O-type extended MTL. Fig. 3(b) shows the creation of a 3-joint P-type extended MTL by replacing the second unit of a basic 3-joint MTL, TU-1, with a P-type unit, TU-6.

Based on Eq. (8), the composition of an extended MTL can be determined. For example, the composition of a six-link 2-joint O-type extended MTL must satisfy the following equation:

$$
6=m_{\mathrm{IU}}+\left.m_{\mathrm{TU}}\right|_{1}-(2-1)
$$

There are two possible unit combination conditions: [(four-link O-type IU), (three-link O-type TU)] and [(two-link O-type IU), (five-link O-type TU)]. For the first set of composition, it can be found from Table 1 that there is one admissible four-link O-type IU and only one three-link Otype TU as well. Hence, the total number of corresponding MTL is one. For the second set of composition, it can be found from Table 1 that there are only one admissible two-link O-type IU and four five-link O-type TUs, including a flippable unit, TU-4. Hence, the total number of 
Table 2

Unit compositions: (a) 1-joint up-to-five link MTLs, (b) 2-joint up-to-seven link MTLs, (c) 3-joint up-to-nine link MTLs

\begin{tabular}{|c|c|c|c|c|c|c|}
\hline & \multirow[t]{2}{*}{$m_{\mathrm{m}}$} & \multicolumn{3}{|c|}{ Number of links } & \multicolumn{2}{|c|}{ Number of MTLs } \\
\hline & & $\overline{\mathrm{IU}}$ & $\mathrm{TU}$ & TU & Subtotal & Total \\
\hline \multicolumn{7}{|l|}{ (a) $\alpha$-type } \\
\hline Basic MIL & 2 & 2 & & & & 1 \\
\hline \multirow[t]{2}{*}{ O-type extended MIL } & 4 & 4 & & & & 1 \\
\hline & 5 & 5 & & & & 1 \\
\hline \multirow[t]{2}{*}{ P-type extended MIL } & 4 & 4 & & & & 2 \\
\hline & 5 & 5 & & & & 2 \\
\hline \multicolumn{7}{|l|}{ (b) $\beta$-type } \\
\hline Basic MIL & 4 & 2 & 3 & & 1 & 1 \\
\hline \multirow[t]{5}{*}{ O-type extended MIL } & 5 & 2 & 4 & & 1 & 1 \\
\hline & 6 & 4 & 3 & & 1 & 5 \\
\hline & & 2 & 5 & & 4 & \\
\hline & 7 & 4 & 4 & & 1 & 2 \\
\hline & & 5 & 3 & & 1 & \\
\hline \multirow[t]{4}{*}{ P-type extended MIL } & 6 & 4 & 3 & & 2 & 6 \\
\hline & & 2 & 5 & & 4 & \\
\hline & 7 & 5 & 3 & & 2 & 4 \\
\hline & & 4 & 4 & & 2 & \\
\hline \multicolumn{7}{|l|}{ (c) $\gamma$-type } \\
\hline Basic MIL & 6 & 2 & 3 & 3 & 1 & 1 \\
\hline \multirow[t]{11}{*}{ O-type extended MIL } & 7 & 2 & 4 & 3 & 1 & 2 \\
\hline & & 2 & 3 & 4 & 1 & \\
\hline & 8 & 4 & 3 & 3 & 1 & 10 \\
\hline & & 2 & 5 & 3 & 4 & \\
\hline & & 2 & 3 & 5 & 4 & \\
\hline & & 2 & 4 & 4 & 1 & \\
\hline & 9 & 5 & 3 & 3 & 1 & 7 \\
\hline & & 4 & 4 & 3 & 1 & \\
\hline & & 4 & 3 & 4 & 1 & \\
\hline & & 2 & 5 & 4 & 4 & \\
\hline & & 2 & 4 & 5 & 4 & \\
\hline \multirow[t]{3}{*}{ P-type extended MIL } & 8 & 4 & 3 & 3 & 2 & 10 \\
\hline & & 2 & 5 & 3 & 4 & \\
\hline & & 2 & 3 & 5 & 4 & \\
\hline
\end{tabular}


Table 2 (continued)

\begin{tabular}{|c|c|c|c|c|c|}
\hline \multirow[t]{2}{*}{$m_{\mathrm{m}}$} & \multicolumn{3}{|c|}{ Number of links } & \multicolumn{2}{|c|}{ Number of MTLs } \\
\hline & $\mathrm{IU}$ & TU & TU & Subtotal & Total \\
\hline 9 & 5 & 3 & 3 & 2 & 14 \\
\hline & 4 & 4 & 3 & 2 & \\
\hline & 4 & 3 & 4 & 2 & \\
\hline & 2 & 5 & 4 & 4 & \\
\hline & 2 & 4 & 5 & 4 & \\
\hline
\end{tabular}

corresponding MTL is four. Table 2 shows the admissible unit combinations with number of links up to $\left.m_{\mathrm{m}}\right|_{\mathrm{b}}$ plus three where $\alpha, \beta$ and $\gamma$ denote 1-, 2-, and 3-joint MTLs, respectively.

\subsection{Speed ratio of mechanical transmission lines}

In Fig. 3(a), the primary links are numbered sequentially as $i$ and $i+1, \ldots, i+n-1$, and $i+n$, and the connecting links are numbered as $j$ and $j+1, \ldots$, and $j+n-1$, respectively. The relation between the local input, $q_{j+n-2, i+n-1}$, and the local output, $q_{i+n, i+n-1}$, of the $n$th unit of the $n$-joint MTL can be related by

$$
q_{j+n-2, i+n-1}=G_{n} q_{i+n, i+n-1}=G_{n} \theta_{i+n}
$$

where $G_{n}$ is the forward gain of the $n$th unit.

Similarly, for the $(n-1)$ th units, we have

$$
q_{j+n-3, i+n-2}=G_{n-1} q_{j+n-2, i+n-2}
$$

From the coaxial condition, Eq. (12) can be rewritten as

$$
q_{j+n-3, i+n-2}=G_{n-1}\left(q_{j+n-2, i+n-1}+\theta_{i+n-1}\right)
$$

By substituting Eq. (11) into Eq. (13), we have

$$
q_{j+n-3, i+n-2}=G_{n-1}\left(G_{n} \theta_{i+n}+\theta_{i+n-1}\right)
$$

Eq. (14) shows the backward propagation of the kinematic relation between adjacent units. From Eq. (14), it can be seen that the angular displacement between the local input and primary link of the $(n-1)$ th unit can be written as function of joint angles succeeding the unit. Hence, the angular displacement of the local input and primary link of the second unit can be written as

$$
q_{j, i+1}=\sum_{m=i+2}^{i+n}\left[\left(\prod_{k=2}^{m-i} G_{k}\right) \theta_{m}\right]
$$

For the first unit, $q_{j+1, i}$ can be written as

$$
q_{j, i}=G_{1}\left(q_{j, i+1}+\theta_{i+1}\right)
$$

By substituting Eq. (15) into Eq. (16), the input angular displacement, $q_{j, i}$, of a $n$-joint MTL can be generally written as a function of joint angles as: 


$$
q_{j, i}=\sum_{m=i+1}^{i+n}\left[\left(\prod_{k=1}^{m-i} G_{k}\right) \theta_{m}\right]
$$

Note that, for basic MTLs, $G_{1}$ is equal to $g_{\mathrm{IU}-1}$ and $G_{2}=G_{3}=\cdots=G_{n}=g_{\mathrm{TU}-1}$.

For the basic 3-joint MTL shown in Fig. 1(d), input angular displacement, $q_{4,0}$, can be written as

$$
\begin{aligned}
q_{4,0} & =g_{\mathrm{IU}-1} \theta_{1}+g_{\mathrm{IU}-1} g_{\mathrm{TU}-1} \theta_{2}+g_{\mathrm{IU}-1} g_{\mathrm{TU}-1} g_{\mathrm{TU}-1} \theta_{3} \\
& =\left[\begin{array}{lll}
1 & e_{5,4} & e_{5,4} e_{3,5}
\end{array}\right]\left[\begin{array}{c}
\theta_{1} \\
\theta_{2} \\
\theta_{3}
\end{array}\right]
\end{aligned}
$$

For the case of extended MTLs, input angular displacement can be also determined by Eq. (17). Hence, input angular displacement, $q_{4,0}$, of the extended MTL shown in Fig. 3(b) can be written as

$$
q_{4,0}=\left[\begin{array}{lll}
1 & \frac{e_{7,6}}{1-e_{1,5} e_{5,6}} & e_{3,7} \frac{e_{7,6}}{1-e_{1,5} e_{5,6}}
\end{array}\right]\left[\begin{array}{c}
\theta_{1} \\
\theta_{2} \\
\theta_{3}
\end{array}\right]
$$

\section{Composition of geared robot manipulators}

From the structural hierarchy, an $n$-DOF GRM is composed of n MTLs by using EOLC as the common linkage as shown in Fig. 1(c). Thus, the GRM shown in Fig. 1(b) can be viewed as the composition of a 1-joint, and two 3-joint MTLs by sharing the primary links as common linkage. Note that there are $n+1$ primary links for an $n$-DOF GRM, while there are $n_{i}+1$ primary links shared with other MTLs as a common linkage for a $n_{i}$-joint MTL. Let $\left.m_{\mathrm{m}}\right|_{i}$ be the number of links of the $i$ th $n_{i}$-joint MTL, the number of links of a $n$-joint GRM, $m_{\mathrm{g}}$, can be represented as

$$
m_{\mathrm{g}}=(n+1)+\sum_{i=1}^{n}\left[\left.m_{\mathrm{m}}\right|_{i}-\left(n_{i}+1\right)\right]
$$

\subsection{Creation of basic geared robot manipulators}

A GRM with only basic MTLs is defined as a basic GRM. The construction of a basic GRM can be treated as the problem of choosing basic MTLs according to the desired configuration of the mechanism.

By substituting Eq. (9) into Eq. (20), the number of links of an $n$-DOF basic GRM, $\left.m_{\mathrm{g}}\right|_{\mathrm{b}}$, can be rewritten as:

$$
\left.m_{\mathrm{g}}\right|_{\mathrm{b}}=(n+1)+\sum_{i=1}^{n}\left(n_{i}-1\right)
$$

For instance, the minimum number of links for a 3-DOF basic GRM with $\alpha \beta \gamma$ configuration can be determined as: 
Table 3

Structure matrices and minimum number of links

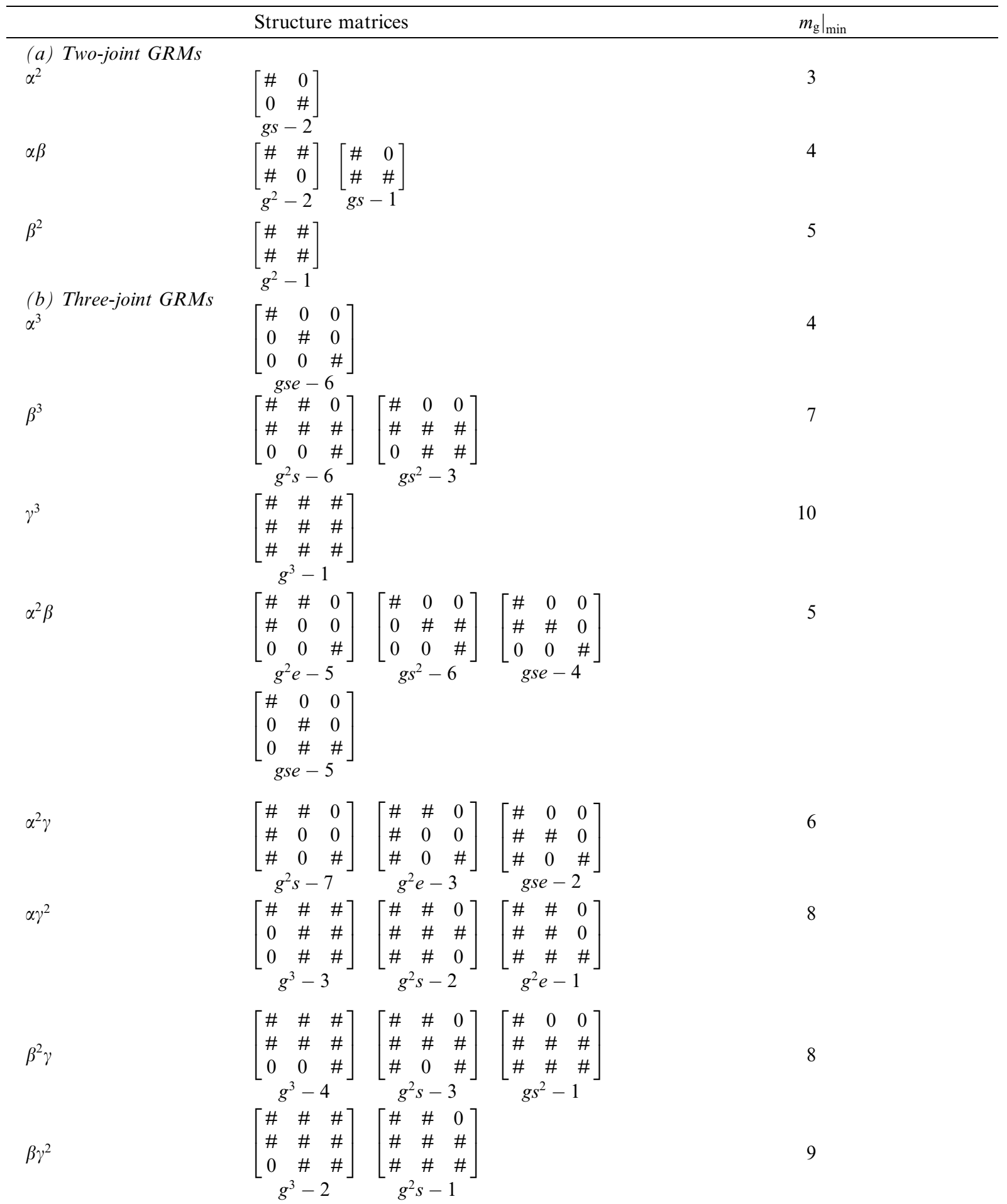


Table 3 (continued)

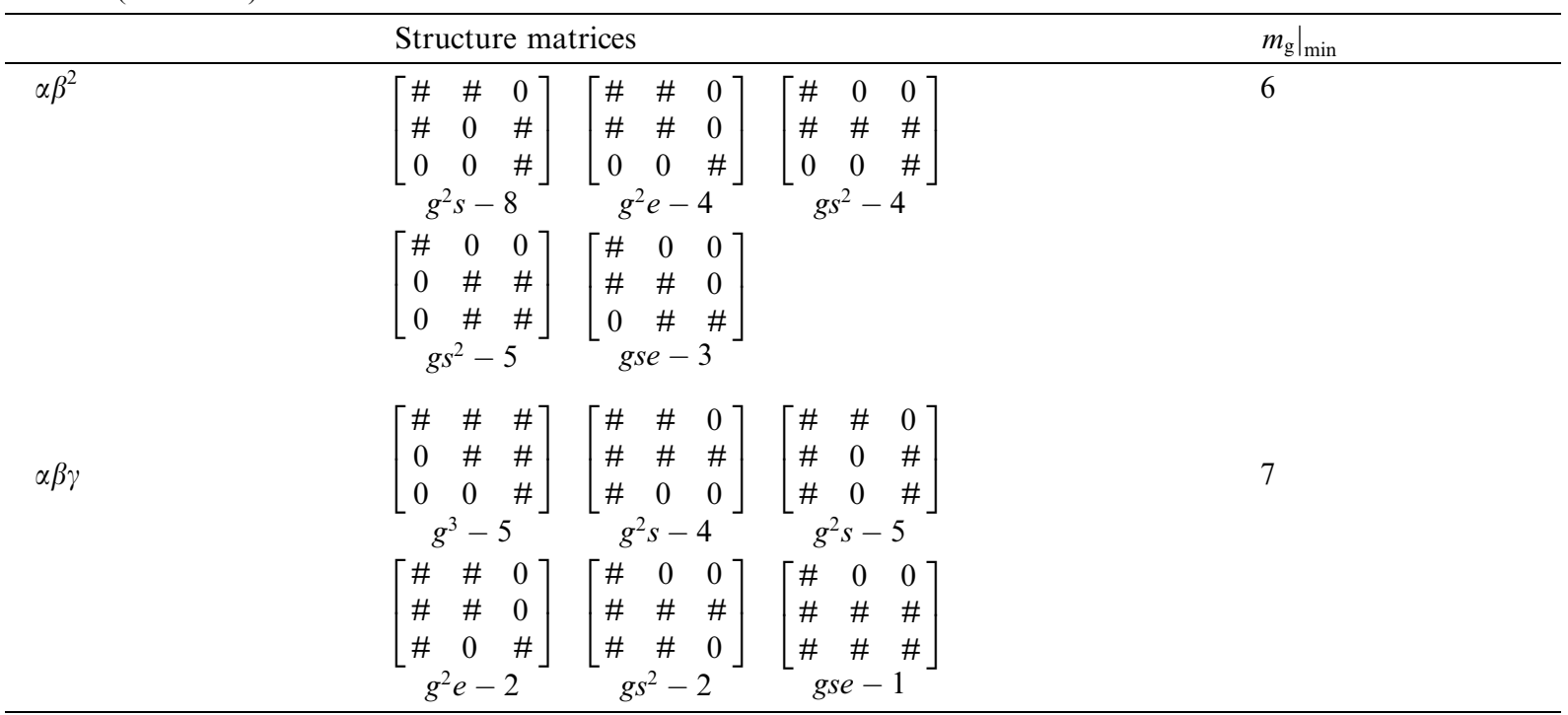

$$
\left.m_{\mathrm{g}}\right|_{\mathrm{b}}=(3+1)+[(3-1)+(2-1)+(1-1)]=7
$$

Note that the number of links for a basic GRM is also the minimum number of links for a GRM with the given configuration. Table 3 shows the configurations, corresponding structure matrices [2] and also the minimum number of links of 2- and 3-DOF GRMs. In Table 3, the orders of $\alpha, \beta$ and $\gamma$ represent the number of associated MTLs. Note that the summation of the orders of $\alpha, \beta$ and $\gamma$ is equal to the number of DOF of the mechanism.

\subsection{Creation of extended geared robot manipulators}

A GRM which has at least one extended MTL is called an extended GRM. Extended GRMs can be obtained by replacing basic MTL(s) in a basic GRM with an extended MTL according to the desired kinematic properties. A GRM with at least one P-type MTL is called a P-type extended GRM while a GRM with only O-type MTLs is called an O-type extended GRM.

Example 1. Two-DOF extended GRMs with $\alpha \beta$ configuration:

From Table 3(a), it can be found that the basic GRM with $\alpha \beta$ configuration has four links. The modification can be carried out as shown in the following illustrative cases:

(1) Relocate the input of the $\alpha$-MTL.

This can be done by replacing the IU-1 unit of MTL with any other O-type input units. As IU-2 is selected, the total number of links of the mechanism can be computed according to Eq. (20) and Table 2 as 


$$
\begin{aligned}
m_{\mathrm{g}} & =3+\left[\left.m_{\mathrm{m}}\right|_{1}-(1+1)\right]+\left[\left.m_{\mathrm{m}}\right|_{2}-(2+1)\right] \\
& =3+(4-2)+(4-3)=6
\end{aligned}
$$

where $\left.m_{\mathrm{m}}\right|_{1}$ and $\left.m_{\mathrm{m}}\right|_{2}$ are the number of links of $\alpha$ and $\beta$ type MTLs, respectively.

Since only O-type units are used, the resultant mechanism can be classified as an O-type extended GRM.

(2) Increase the forward gain at the second joint of the mechanism.

This can be done by replacing the TU-1 unit of the $\beta$-MTL with any other P-type transmission units. As TU-6 is selected, the total number of links of the mechanism can be computed according to Eq. (20) and Table 2 as

$$
\begin{aligned}
m_{\mathrm{g}} & =3+\left[\left.m_{\mathrm{m}}\right|_{1}-(1+1)\right]+\left[\left.m_{\mathrm{m}}\right|_{2}-(2+1)\right] \\
& =3+(2-2)+(6-3)=6
\end{aligned}
$$

Since a P-type unit is used, the resultant mechanism can be classified as a P-type extended GRM. Table 4 shows the complete result of admissible MTL combinations for GRMs with $\alpha \beta$ configuration with up-to-six links.

Example 2. Three-DOF P-type extended GRMs with $\alpha \beta \gamma$ configuration:

From Table 3(a), it can be found that the basic GRM with $\alpha \gamma$ configuration has seven links. According to required kinematic behavior, the modification can be carried out as shown in the following illustrative cases:

(1) Increase the span of the second joint.

One possible way to achieve the design requirement is to replace both the only TU-1 unit of the $\beta$-MTL and the second TU- 1 unit of the $\gamma$-MTL with other O-type transmission units. As TU-2 is selected to replace the two TU- 1 units, the arrangements of the units in the $\beta$-MTL and $\gamma$-MTL

\begin{tabular}{|c|c|c|c|c|c|c|}
\hline \multirow[t]{2}{*}{$\mathbf{A}$} & \multirow[t]{2}{*}{ Type } & \multirow[t]{2}{*}{$m_{\mathrm{g}}$} & \multicolumn{2}{|c|}{$m_{\mathrm{m}}$} & \multirow{2}{*}{\multicolumn{2}{|c|}{$\begin{array}{l}\text { Admissible mechanisms } \\
\text { total }\end{array}$}} \\
\hline & & & $\bar{\alpha}$ & $\beta$ & & \\
\hline \multirow[t]{5}{*}{$\alpha \beta$} & Basic GRM & 4 & 2 & 4 & 1 & 1 \\
\hline & O-type Extended GRM & 5 & 2 & 5 & 1 & 1 \\
\hline & & 6 & $\begin{array}{l}2 \\
4\end{array}$ & $\begin{array}{l}6 \\
4\end{array}$ & $\begin{array}{l}5 \\
1\end{array}$ & 6 \\
\hline & P-type Extended GRM & 6 & 2 & 6 & 6 & 8 \\
\hline & & & 4 & 4 & 2 & \\
\hline
\end{tabular}
can be expressed as

Table 4

Admissible MTL combinations of up-to-six link GRMs with $\alpha \beta$ configuration 
$\alpha$-MTL: $\quad[\mathrm{IU}-1]$

$\beta$-MTL: $[\mathrm{IU}-1, \mathrm{TU}-2]$

$\gamma$-MTL: $[$ IU-1, TU-2, TU-1]

and the number of links of the mechanism can be computed according to Eq. (20) and Table 2 as

$$
\begin{aligned}
m_{\mathrm{g}} & =(3+1)+\left[\left.m_{\mathrm{m}}\right|_{1}-(1+1)\right]+\left[\left.m_{\mathrm{m}}\right|_{2}-(2+1)\right]+\left[\left.m_{\mathrm{m}}\right|_{3}-(3+1)\right] \\
& =4+(2-2)+(5-3)+(7-4)=9
\end{aligned}
$$

where $\left.m_{\mathrm{m}}\right|_{1},\left.m_{\mathrm{m}}\right|_{2}$ and $\left.m_{\mathrm{m}}\right|_{3}$ are the number of links of $\alpha, \beta$ and $\gamma$ MTLs, respectively.

The resultant mechanism is an O-type extended GRM since the units for replacement are of Otype.

(2) Increase the forward gain at the first joint of the mechanism.

This can be done by replacing the basic input units in any one of the three MTLs with a P-type transmission unit. As IU-3 is selected to replace the IU- 1 unit in the $\beta$-MTL, the arrangements of the units can be expressed as

$$
\begin{array}{ll}
\alpha \text {-MTL: } & {[\mathrm{IU}-1]} \\
\beta \text {-MTL: } & {[\mathrm{IU}-3, \mathrm{TU}-1]} \\
\gamma \text {-MTL: } & {[\mathrm{IU}-1, \mathrm{TU}-1, \mathrm{TU}-1]}
\end{array}
$$

and the number of links of the mechanism can be computed according to Eq. (20) and Table 2 as

$$
\begin{aligned}
m_{\mathrm{g}} & =(3+1)+\left[\left.m_{\mathrm{m}}\right|_{1}-(1+1)\right]+\left[\left.m_{\mathrm{m}}\right|_{2}-(2+1)\right]+\left[\left.m_{\mathrm{m}}\right|_{3}-(3+1)\right] \\
& =4+(2-2)+(6-3)+(6-4)=9
\end{aligned}
$$

\begin{tabular}{|c|c|c|c|c|c|c|c|}
\hline \multirow[t]{2}{*}{$\mathbf{A}$} & \multirow[t]{2}{*}{ Type } & \multirow[t]{2}{*}{$m_{\mathrm{g}}$} & \multicolumn{3}{|c|}{$m_{\mathrm{m}}$} & \multirow{2}{*}{\multicolumn{2}{|c|}{$\begin{array}{l}\text { Admissible } \\
\text { mechanisms subtotal }\end{array}$}} \\
\hline & & & $\bar{\alpha}$ & $\beta$ & $\gamma$ & & \\
\hline \multirow[t]{10}{*}{$\alpha \beta \gamma$} & Basic GRM & 7 & 2 & 4 & 6 & 1 & 1 \\
\hline & O-type Extended GRM & 8 & 2 & 5 & 6 & 1 & 3 \\
\hline & & & 2 & 4 & 7 & 2 & \\
\hline & & 9 & 4 & 4 & 6 & 1 & 18 \\
\hline & & & 2 & 5 & 7 & 2 & \\
\hline & & & 2 & 6 & 6 & 5 & \\
\hline & & & 2 & 4 & 8 & 10 & \\
\hline & P-type Extended GRM & 9 & 4 & 4 & 6 & 2 & 18 \\
\hline & & & 2 & 6 & 6 & 6 & \\
\hline & & & 2 & 4 & 8 & 10 & \\
\hline
\end{tabular}

The resultant mechanism is a P-type extended GRM since a P-type input unit is used. Table 5 shows the complete result of admissible MTL combinations of up-to-nine link GRMs with $\alpha \beta \gamma$

Table 5

Admissible MTL combinations of up-to-nine link GRMs with $\alpha \beta \gamma$ configuration 

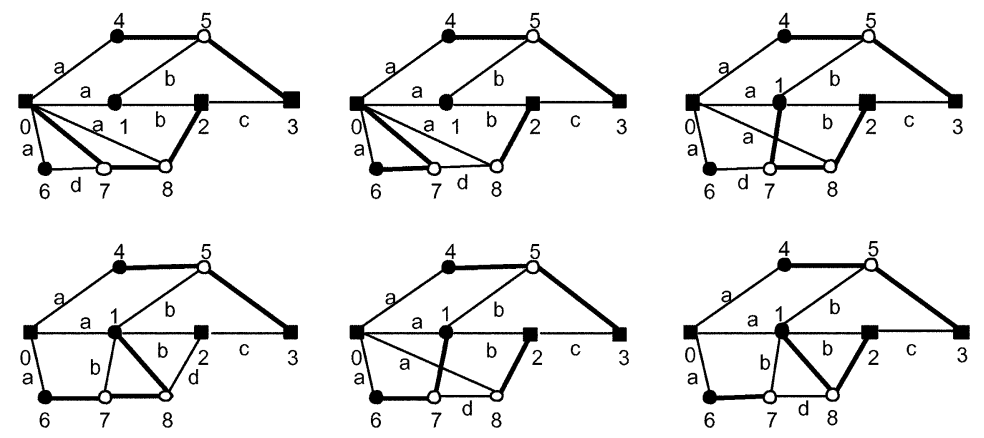

Fig. 4. Admissible graphs of 3-DOF nine-link GRMs.

configuration. Those GRMs with $\left[\left.m_{\mathrm{m}}\right|_{1},\left.m_{\mathrm{m}}\right|_{2},\left.m_{\mathrm{m}}\right|_{3}\right]=[2,6,6]$ are shown in Fig. 4 in which the first one represents the mechanism created in above case.

After the arrangement of units in each MTL has been determined, the kinematic relation among the inputs and each articulated joint can be obtained by combining the propagation results of each MTL, which can be derived from Eq. (17). For the first GRM shown in Fig. 4, input angular displacement of the direct-drive two-link MTL, $q_{1,0}$, can be written as

$$
q_{1,0}=\theta_{1}
$$

From Eq. (17), input angular displacement $q_{6,0}$ can be determined as

$$
q_{6,0}=\left[\begin{array}{lll}
\frac{1}{1-e_{077} 78} & e_{28} \frac{1}{1-e_{077} 78} & 0
\end{array}\right]\left[\begin{array}{l}
\theta_{1} \\
\theta_{2} \\
\theta_{3}
\end{array}\right]
$$

By combining Eqs. (18), (27) and (28), the complete relation between the joint space and the actuator space can be expressed as

$$
\left[\begin{array}{l}
q_{1,0} \\
q_{6,0} \\
q_{4,0}
\end{array}\right]=\left[\begin{array}{ccc}
1 & 0 & 0 \\
\frac{1}{1-e_{07} e_{78}} & e_{28} \frac{1}{1-e_{07} e_{78}} & 0 \\
1 & e_{54} & e_{54} e_{35}
\end{array}\right]\left[\begin{array}{l}
\theta_{1} \\
\theta_{2} \\
\theta_{3}
\end{array}\right]
$$

From Eq. (29), it can be seen that by adjusting the value of $e_{70} e_{87}$ to be close enough to unity, the input of the $\beta$-MTL can significantly magnified at the first joint to achieve the design requirement.

\section{Conclusion}

In this paper, a methodology for the modular design of GRMs in the conceptual design stage is presented. Based on the hierarchical structure, a GRM can be viewed as a combination of input and transmission units which can be further categorized into modular groups according to their number of links and kinematic characteristics. Hence, in the conceptual design stage, basic GRMs can be enumerated first by composing basic MTLs, which contains only basic units according to the given configuration. Modified structures are then obtained by replacing the contained basic 
unit(s) with extended unit(s) in the modular groups to meet design specifications such as center distance, speed ratio, and relocation of actuators. This approach allows a designer to acquire proper design(s) from the simplest mechanical structure during the conceptual design stage.

\section{References}

[1] C.C. Lin, L.W. Tsai, The development of an atlas of bevel-gear-type spherical wrist mechanisms, in: Proceedings of the First National Conference on Applied Mechanisms and Robotics, paper no. 89-AMR-2A-3, 1989.

[2] S.L. Chang, L.W. Tsai, Topological synthesis of articulated gear mechanisms, IEEE Transactions on Robotics and Automation 6 (1) (1990) 97.

[3] D.Z. Chen, S.C. Shiue, Topological synthesis of geared robotic mechanism, ASME Journal of Mechanical Design 120 (1998) 230.

[4] F. Freudenstein, An application of boolean algebra to the motion of epicyclic drives, ASME Journal of Engineering for Industry 93 (B) (1971) 176.

[5] L.W. Tsai, An application of the linkage characteristic polynomial to the topological synthesis of epicyclic gear trains, ASME Journal of Mechanisms Transmissions, and Automation in Design 109 (3) (1987) 329.

[6] R. Ravisankar, T.S. Mruthyunjaya, Computerized synthesis of the structure of geared kinematic chains, Mechanism and Machine Theory 20 (1985) 367.

[7] J.T. Kim, B.M. Kwak, Application of edge permutation group to structural synthesis of epicyclic gear trains, Mechanism and Machine Theory 25 (5) (1990) 563.

[8] L.W. Tsai, The kinematics of spatial robotic bevel-gear trains, IEEE Journal of Robotics and Automation 4 (2) (1988) 150.

[9] D.Z. Chen, Gear train configuration arrangement of articulated gear mechanisms based on the generalized principle of inertia match, Journal of the Chinese Society of Mechanical Engineers 16 (1) (1995) 29.

[10] D.Z. Chen, Kinematic analysis of geared robot manipulators by the concept of structural decomposition, Mechanism and Machine Theory 33 (7) (1998) 975.

[11] D.Z. Chen, C.P. Liu, A hierarchical decomposition scheme for the topological synthesis of articulated gear mechanisms, ASME Journal of Mechanical Design 121 (2) (1999) 256. 